\title{
Evaluation of black glutinous rice (Oryza sativa L) extract as a novel nuclear stain for human sperm head assessment by microscopic examination
}

\author{
Sirinart Chomean 1 , Tanawan Sukanto' , Arreya Piemsup ${ }^{1}$, Jirattikan Chaiya ${ }^{2}$, Kolunya Saenguthai $^{3}$, Chollanot Kaset ${ }^{1}$ \\ 'Department of Medical Technology, Faculty of Allied Health Sciences, Thammasat University, Pathumthani; ${ }^{2}$ Thammasat Infertility Center and ${ }^{3}$ Medical \\ laboratory, Thammasat University Hospital, Thammasat University, Pathumthani, Thailand
}

Objective: To compare black rice (Oryza sativa L) extract with three different staining methods for human sperm head assessment.

Methods: Semen samples were collected from 34 volunteers. Four smears of each ejaculate were prepared for staining using the rapid Papanicolaou (PAP) stain, SpermBlue, DipQuick, and black rice extract. The percentage of defective sperm heads (mean \pm standard deviation) was compared.

Results: Black glutinous rice extract, a natural dye, was used instead of hematoxylin to stain the nuclei of the sperm heads. The percentage of defective sperm heads showed a significant difference between black rice extract and DipQuick $(p=0.000)$. In contrast, black rice extract and rapid PAP showed no statistically significant difference $(p=0.974)$. A strong correlation $(r=0.761)$ was found between the findings obtained using rapid PAP and black rice extract. In contrast, a weak correlation $(r=0.248)$ was obtained between DipQuick and black rice extract for the percentage of defective sperm heads.

Conclusion: The results showed good agreement and a strong correlation between the rapid PAP and black rice extract stains. The advantages of black rice extract as a novel substitute for hematoxylin for nuclear staining include ease of preparation, local availability, and favorable nuclear staining properties. Further studies could also focus on comparing staining techniques in clinical samples.

Keywords: Black glutinous rice; Microscopic examination; Papanicolaou; Rapid staining; Sperm head

\section{Introduction}

For the evaluation of sperm morphology, the World Health Organization (WHO) has recommended standard staining procedures, including the Papanicolaou (PAP) stain, the Shorr stain, and rapid stain-

Received: Feb 20, 2019 · Revised: Apr 2, 2019 Accepted: Apr 2, 2019

Corresponding author: Chollanot Kaset

Department of Medical Technology, Faculty of Allied Health Sciences,

Thammasat University, Rangsit Campus, 99 Moo18 Phahonyothin Road, Khlong Nueng, Khlong Luang, Pathum Thani 12120, Thailand

Tel: +66-2986-9213-9 (\#7225-6) Fax:+66-2516-5379 E-mail:chollanotk@gmail.com

* This study was supported by the Faculty of Allied Health Sciences and Thammasat University Research Fund (Contract No. 1/2560).

This is an Open Access article distributed under the terms of the Creative Commons Attribution Non-Commercial License (http://creativecommons.org/licenses/by-nc/4.0/) which permits unrestricted non-commercial use, distribution, and reproduction in any medium, provided the original work is properly cited. ing (DiffQuik) [1]. Although DiffQuik causes sperm swelling and background staining [2], it is still used as a routine method for manual and computer-assisted sperm analysis (CASA) $[2,3]$. The DiffQuik involves a short fixation step followed by eosin and methylene blue as a nuclear stain, with each step taking a few seconds. A new stain, SpermBlue, has been developed for the evaluation of sperm morphology $[4,5]$. The main advantage of SpermBlue is that it provides a simple and fast staining method. Furthermore, the fixative and stain components of SpermBlue are iso-osmotic with human semen, meaning that SpermBlue has no significant effect on sperm head dimensions [5]. In contrast, the PAP staining method, which is used for cervical cancer screening in gynecology, involves more than 20 processing steps with more than 12 different chemical solvents [1], making it time-consuming. Moreover, the fixation and dehydration steps may also cause sperm shrinkage [5]. 
A natural dye, hematoxylin is an essential component of the PAP stain and is used in many hemalum staining solutions that provide blue staining in cell nuclei. Dapson et al. [6] reported a shortage of hematoxylin in the first half of 2008. Due to the resulting uncertainties, an alternative nuclear stain is needed. The red and purple colors of various flowers, rice, and fruit are due to anthocyanin. Asian countries account for almost 95\% of rice production [7], and there are some specialty rice strains with distinct colors due to the deposition of large amounts of anthocyanin pigment in the rice coat [8]. Black glutinous rice contains a large amount of anthocyanin, a pigment that is a water-soluble flavonoid [8]. Natural pigments have been studied, and a combination of roselle (Hibiscus sabdariffa) with ferric chloride followed by eosin dye was found to give a histological picture similar to that provided by hematoxylin and eosin [9].

Because the frequency of sperm head defects is much higher than the frequency of defects in other parts of sperm cells, the sperm head assessment can significantly affect the results of a sperm morphology assessment [10]. The objective of this study was to compare three different staining methods with a novel black rice (Oryza sativa L) extract as a nuclear stain substitute for hematoxylin in an assessment of sperm head morphology using manual microscopic examinations.

\section{Methods}

\section{Sample}

Semen samples were collected from 34 reproductive-age volunteers (18-35 years) at Thammasat University. A consent form was signed by each donor participating in this study according to regulation No. 077/2560 of the third ethical committee of Thammasat University. Human sperm donor selection was based on semen volume ( $\geq 1.5 \mathrm{~mL}$ ) and sperm concentration ( $\geq 15 \times 10^{6} / \mathrm{mL}$ ) [1].

\section{Slide preparation}

A $10-\mu \mathrm{L}$ sample was smeared on a precleaned glass slide. Four smears of each ejaculate were prepared. For rapid PAP staining, smeared slides were fixed in 95\% ethanol for 1 minute, and then were transferred to hematoxylin for 15 minutes, followed by differentiation with $1 \%$ acid alcohol for 10 dips. Subsequently, the slides were dipped into bluing solution in tap water 10 times, followed by polychome mixture (Eosin Azure consists of three dyes: Eosin Y, light green SF yellowish and Bismarck brown Y. ) with orange $\mathrm{G}$ for $15 \mathrm{~min}$ utes. Dehydration was done with two changes of dehydration buffer. For the DipQuick method (identical to the DiffQuik method), the semen smeared slides were fixed in methanol for 1 minute and then were dipped into eosin 10 times, followed by dipping into methylene blue for 10 times. Finally, the slides were washed in running tap wa- ter. The recently-developed SpermBlue (Microptic, Barcelona, Spain) were was also used as a comparator in this study. The air-dried smears were carefully placed vertically into the SpermBlue fixative/ stain for 60 seconds. Subsequently, the slides were dipped slowly in a Coplin jar containing tap water twice for 3 seconds each.

\section{Extraction of dye from black glutinous rice ( 0 . sativa $\mathrm{L}$ ) and staining procedure}

For black rice extraction, $100 \mathrm{~g}$ of black glutinous rice was extracted with $100 \mathrm{~mL}$ of buffer containing $10 \mathrm{~g}$ of potassium alum in $100 \mathrm{~mL}$ of distilled water with $4 \%$ ethanol and $5 \%$ acetic acid. The flasks were then wrapped with aluminum foil and left overnight. The dark purple supernatant was filtered with Whatman filter paper and kept in a dark place until use.

In the black rice extract staining procedure, the smeared slides were fixed in 95\% ethanol for 1 minute and then were transferred to black rice extract for 15 minutes, followed by differentiation with $1 \%$ acid alcohol 10 times. Subsequently, slides were dipped into bluing solution in tap water 10 times, followed by polychrome-ORG for 15 minutes to stain the cytoplasm part of the sperm. Dehydration was done with two changes of dehydration buffer.

\section{Evaluation of sperm head defects}

The spermatozoa head defect assessment was conducted by five different observers, including an assistant professor with 10 years of experience in semen analysis and four scientists from the in vitro fertilization center of Thammasat University Hospital with 10-12 years of experience in semen analysis. The assessment was performed according to the $2010 \mathrm{WHO}$ guideline [1]; a sperm was considered to have a head defect when it had a large or small oval head, a tapering pyriform and vacuolated head, the absence of a acrosome, a double head, or a head with an irregular form. Two hundred spermatozoa from each slide were evaluated to identify the percentage of sperm with a head defect with a bright-field microscope (DP21-Standalone System; Olympus, Waltham, MA, USA) under $\times 100$ magnification.

\section{Statistical analysis}

Statistical analysis was performed using MedCal ver. 18.2.1 (MedCalc, Mariakerke, Belgium) and IBM SPSS ver. 22.0 (IBM Corp., Armonk, NY, USA). Intraclass correlation coefficients (ICCS) were used to assess the reliability index in intrarater and interrater reliability analyses. Two-way random effects, consistency and absolute agreement, and a multiple-raters model were used in this study based on a mean-rating ( $k=5)$. Koo and Li [11] suggested that ICC values less than 0.5 indicate poor reliability, values between 0.5 and 0.75 indicate moderate reliability, values between 0.75 and 0.9 indicate good reliability, and values greater than 0.90 indicate excellent reliability. 
The four methods were compared using the Tukey test. Bland and Altman analysis was used to determine the systematic biases when the differences between two methods were plotted against the average of two methods. Passing-Bablok regression was performed to describe a linear regression with no special assumptions referring to the distribution of the samples and the measurement error. The slope was calculated with a 95\% confidence interval (Cl). The Spearman rank correlation coefficient was used to measure the strength of the monotonic relationship between paired data. The interpretation of correlation coefficients was assessed using five levels: very weak $(0.00<r \leq 0.19)$, weak $(0.20<r \leq 0.39)$, moderate $(0.40<r \leq 0.59)$, strong $(0.60<r \leq 0.79)$, and very strong $(0.80<r \leq 1.00)$. Mountain plots were generated by calculating the percentile of each ranked difference variable for the four staining methods.

Table 1. Intraclass correlation coefficients calculated using two-way random effects, consistency and absolute agreement, and multiple rating models

\begin{tabular}{lccc}
\hline Staining method & $\begin{array}{c}\text { Intraclass } \\
\text { correlation }\end{array}$ & $95 \% \mathrm{Cl}$ & Sig \\
\hline DipQuick & $0.534^{\mathrm{a}}$ & $0.247-0.736$ & 0.000 \\
& $0.653^{\mathrm{b}}$ & $0.428-0.808$ & 0.000 \\
Rapid PAP & $0.654^{\mathrm{a}}$ & $0.365-0.819$ & 0.000 \\
& $0.791^{\mathrm{b}}$ & $0.655-0.884$ & 0.000 \\
SpermBlue & $0.599^{\mathrm{a}}$ & $0.325-0.779$ & 0.000 \\
& $0.715^{\mathrm{b}}$ & $0.530-0.843$ & 0.000 \\
Black rice extract & $0.682^{\mathrm{a})}$ & $0.334-0.847$ & 0.000 \\
& $0.861^{\mathrm{b}}$ & $0.771-0.923$ & 0.000 \\
\hline
\end{tabular}

$\mathrm{Cl}$, confidence interval; PAP, Papanicolaou.

${ }^{\text {a) }}$ Absolute agreement; ${ }^{\text {b) }}$ Consistency agreement.

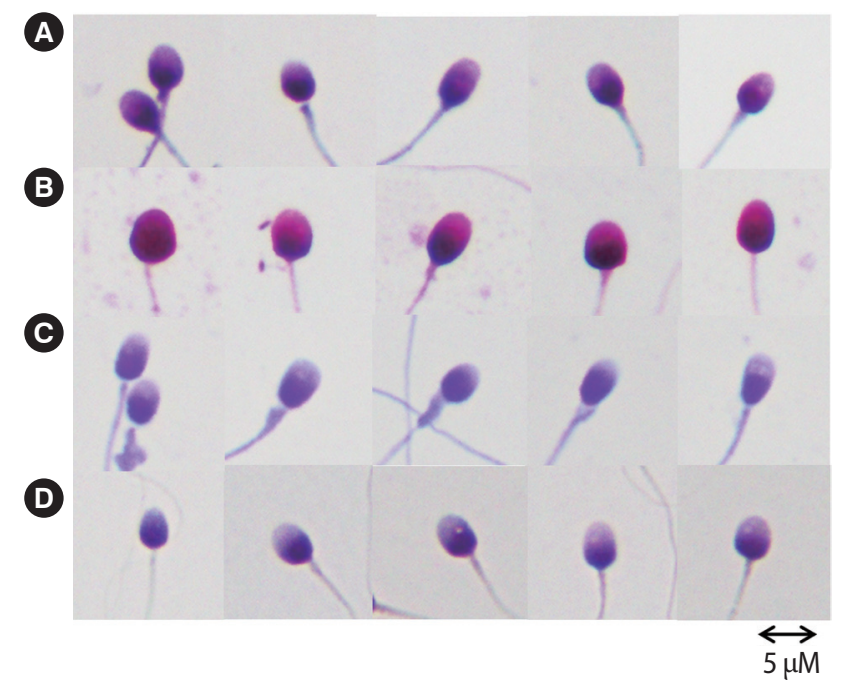

Figure 1. Patterns of sperm morphology after staining with $(A)$ rapid Papanicolaou, (B) DipQuick, (C) SpermBlue, and (D) black rice extract. The scale bars of all panels were set at $5 \mu \mathrm{M}$.

\section{Results}

ICCs were used to analyze the reliability index for intrarater and interrater reliability. As shown in Table 1, DipQuick (0.653) and SpermBlue (0.715) showed a moderate level of consistency, whereas rapid PAP and black rice extract showed higher levels of consistency $(0.791$ and 0.861 , respectively), meaning that the agreement between any two of five observers was good. To analyze observer reliability (Table 1), ICC estimates and their $95 \% \mathrm{Cls}$ were calculated based on a mean rating $(k=5)$, absolute agreement, and a two-way random effects model. The ICCs of absolute agreement for DipQuick (0.534) and SpermBlue (0.599) were lower than those for rapid PAP (0.654) and black rice extract (0.682). All of these values indicate a moderate level of absolute agreement.

Figure 1 shows the staining patterns for the four different staining methods used in this study. The acrosome was clear on rapid PAPstained smears (Figure 1A). The head was stained red-purple. The middle piece and tail morphology were identifiable (Figure 1A). Using DipQuick, the spermatozoa were stained dark purple (Figure 1B). The head morphology and acrosome were seen (Figure 1B). The middle piece and tail were seen clearly and could be evaluated, as they showed a pale red color from eosin staining (Figure 1B). In the spermatozoa stained with SpermBlue, head condensation and the midpiece were shown by a purple color, and the tail also was shown by a purple color (Figure 1C). In this study, a natural dye was extracted from black rice, which was used instead of hematoxylin to stain the nuclei of the sperm cells. In the results of this procedure, acrosome condensation and head morphology were clearly visible. The middle piece and tail were identified with polychrome-ORG dye (Figure 1D).

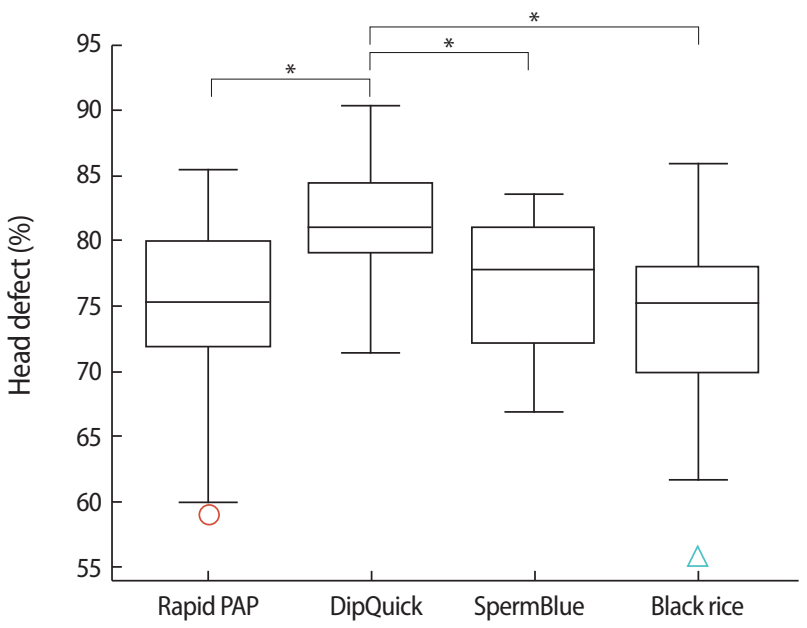

Figure 2. Box and whisker plot demonstrating the percentage of defective sperm heads after staining with different methods $(n=34)$. Circle and triangle are outliers of data. *Significantly different $(p<0.05)$. 
Calculating the mean percentage of sperm with a head defect (Figure 2) yielded the following results: rapid PAP, 74.89\% $\pm 6.24 \%$; DipQuick, 81.33\% \pm 4.25\%; SpermBlue, 76.77\% $\pm 4.90 \%$; and black rice extract, $74.50 \% \pm 6.71 \%$. The most significant difference was between black rice extract and DipQuick $(p=0.000)$. In contrast, there was no statistically significant difference between the rapid PAP and

\section{A}

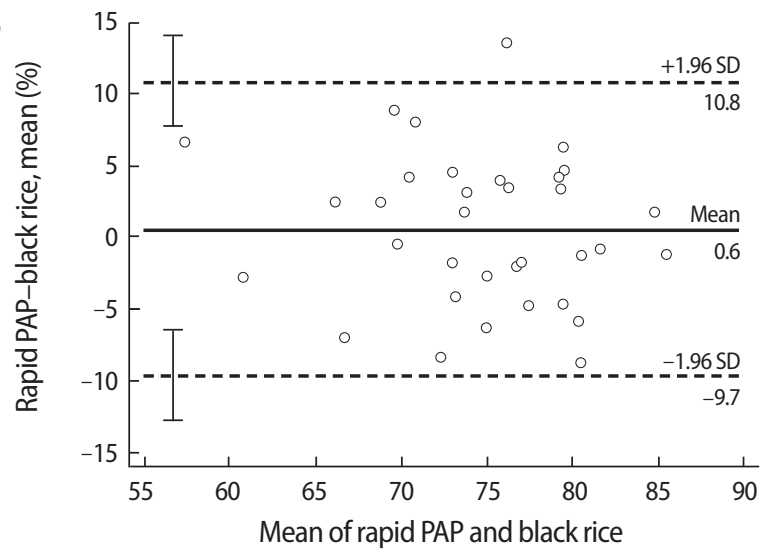

B

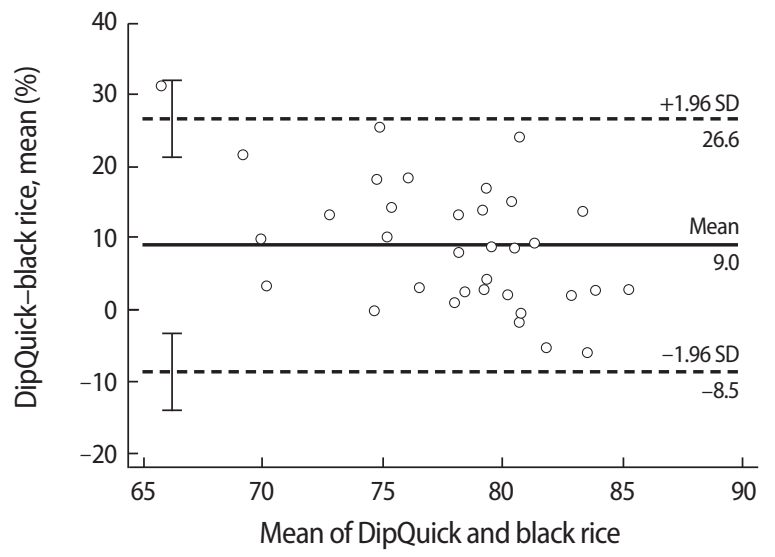

C

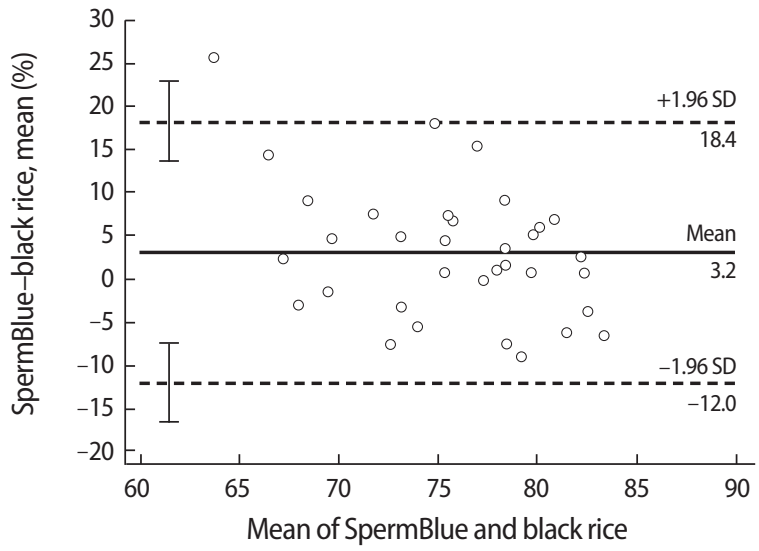

black rice extract staining methods ( $p=0.974)$, and marginal significance was found for the difference between black rice extract and SpermBlue $(p=0.063)$.

Figure 3A-C shows Bland and Altman plots for sperm head defects, which were created after checking that the value of differences was normally distributed. To assess the degree of agreement, Bland and

(D)

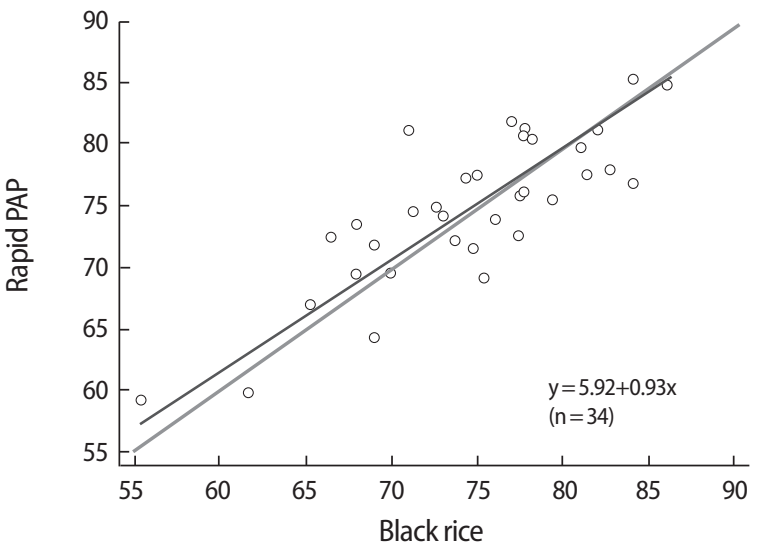

E

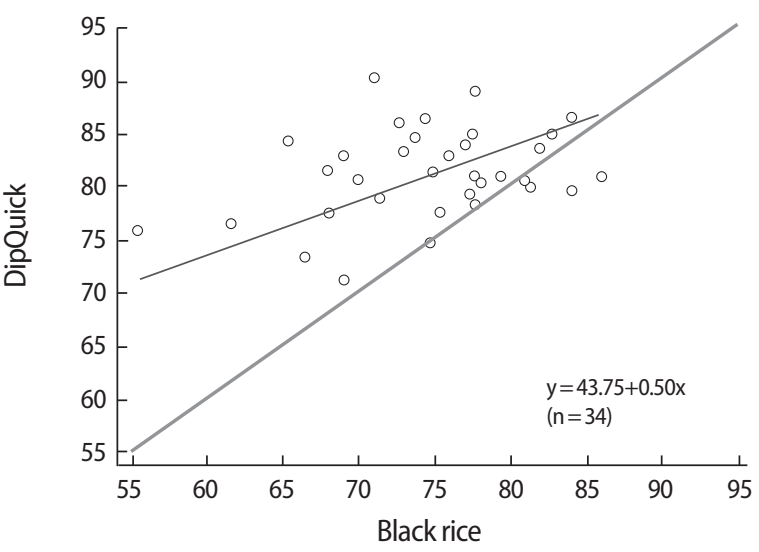

$\boldsymbol{F}$

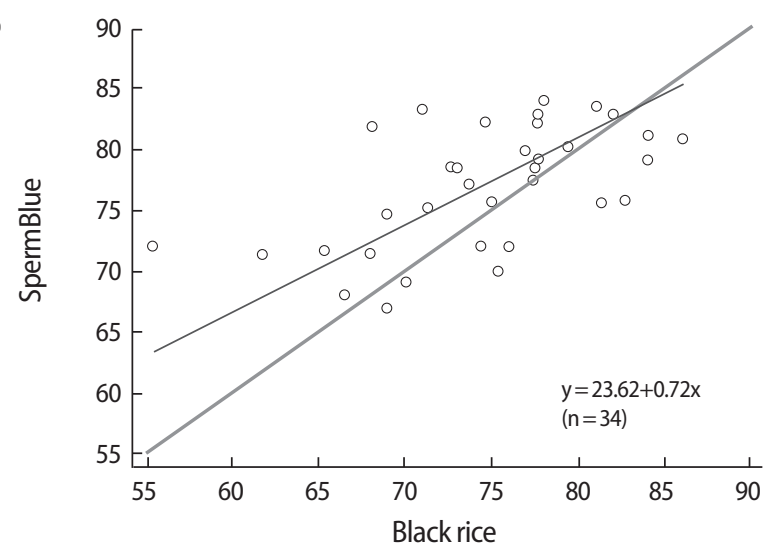

Figure 3. Bland and Altman plots comparing the mean percentage of defective sperm heads after staining with black rice extract and the three staining methods (A-C). The continuous line (bias) represents the mean difference, and the dashed lines represent the $95 \%$ limits of agreement ( \pm 1.96 standard deviations [SDs]). Passing-Bablok regression analyses were conducted to compare pairs of methods for sperm head defect evaluation (D-F). PAP, Papanicolaou. 
Altman analysis was used to qualify the agreement between two quantitative measurements by computing the mean difference and limits of agreement. In the comparison of rapid PAP with the black rice extract stain for head defect evaluation (Figure $3 \mathrm{~A}$ ), the difference was close to zero (mean difference, $0.6 \% \pm 10.2 \%$ ). This result indicates that the mean percentage of defective sperm heads observed after staining with the black rice extract was similar to that observed with rapid PAP. In the comparison of the black rice extract stain with SpermBlue (Figure 3C), the percentage of defective sperm heads determined using SpermBlue was higher than that determined using the black rice extract stain (mean difference, 3.2\% \pm $15.2 \%)$. Conversely, an overestimation of the percentage of defective sperm heads was generated (Figure $3 \mathrm{~B}$ ) when black rice extract was compared with the DipQuick stain (mean difference, 9.0\% $\pm 17.6 \%$ ).

To further compare the different staining methods, Passing-Bablok regression was performed. Figure $3 \mathrm{D}$ shows the regression line between the rapid PAP and black rice extract stains. The resulting equation was: $y=5.92+0.93 x$; the $95 \% \mathrm{Cl}$ was -11.67 to 22.32 for the intercept and 0.70 to 1.16 for the slope, indicating good agreement. The cusum test for linearity indicated no significant deviation from linearity. Similarly, the regression analysis between SpermBlue and the black rice extract stain revealed good agreement and the cusum test for linearity also indicated that there was no significant deviation from linearity ( $p=1.00$; regression line equation: $y=23.62+0.72 x$; $95 \% \mathrm{Cl},-3.00$ to 42.41 for the intercept and 0.46 to 1.06 for the slope) (Figure 3F). In contrast, the regression line equation for the comparison of DipQuick and black rice extract was $y=43.75+0.49 x(95 \% \mathrm{Cl}$, 11.11 to 63.60 for the intercept and 0.23 to 0.94 for the slope, indicating a very large proportional difference). The cusum test for linearity revealed no significant deviation from linearity $(p=0.42)$ (Figure 3E).

The strength of the relationship between pairs of methods for the percentage of defective sperm heads was determined by the Spearman rank correlation coefficient. A strong correlation ( $r=0.761)$ was found between rapid PAP and black rice extract. Likewise, a moderate correlation ( $r=0.588)$ was observed between SpermBlue and black rice extract. However a weak correlation $(r=0.248)$ was found between DipQuick and black rice extract for the percentage of defective sperm heads.

To characterize the distribution of differences between the methods, mountain plots were generated. The difference between the rapid PAP and black rice extract stains tended to be smaller than the difference between rapid PAP and DipQuick. Moreover, the median of the differences was closer to zero for the black rice extract method than for the DipQuick method, as compared with the rapid PAP stain. Thus, the black rice extract stain corresponded better to rapid PAP than the DipQuick method. In contrast, a comparison of SpermBlue and the black rice extract stain with the rapid PAP stain (Figure 4B)
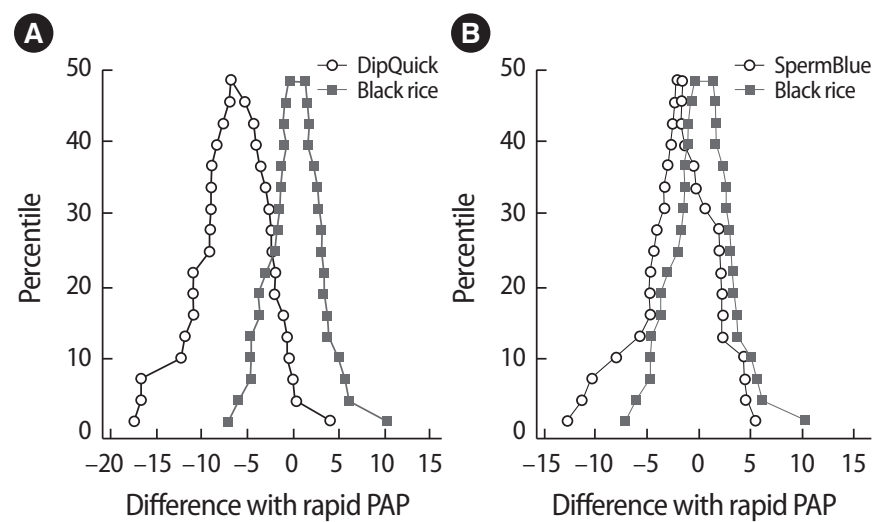

Figure 4. Mountain plots of the DipQuick and black rice extract method with the rapid Papanicolaou (PAP) method (A); the SpermBlue and black rice extract method with the rapid PAP method (B).

showed a close correspondence, suggesting that the results of both methods were similar.

\section{Discussion}

In this study, black rice ( $O$. sativa $\mathrm{L}$ ) extract was used as a novel nuclear stain instead of hematoxylin. The use of anthocyanin from roselle ( $H$. sabdariffa) for staining of lymph node sections has been reported [9]. In recent studies, black rice extract has been used as an alternative to hematoxylin to detect spermatozoa on clothing and vaginal swab casework samples [12]. Moreover, black rice extract has been revealed to provide an interesting alternative to the rapid PAP stain used for spermatozoa assessment [13].

Because the frequency of sperm head defects is much higher than the frequency of defects in other parts of sperm, the sperm head assessment can significantly affect the results of a sperm morphology assessment [10]. The objective of this study was to compare three different staining methods with a novel black rice extract in an assessment of sperm head morphology.

Before assessment staining methods can be used for research or clinical applications, their reliability should be evaluated. The ICC is a useful estimate of reliability that reflects degrees of both correlation and agreement between observers. All staining methods showed ICC values for the absolute agreement between 0.5 and 0.75 , indicating moderate reliability. The ICC values showed good consistency agreement for the rapid PAP and black rice extract stains, but a moderate level of agreement for the DipQuick and SpermBlue.

When a new staining method is innovated, it should be compared with validated staining methods [5]. Black rice extract was compared with three different staining methods (rapid PAP, SpermBlue, and DipQuick) for human sperm head evaluation using the 2010 WHO criteria. After black rice staining, the staining patterns of spermatozoa 
clearly showed acrosome condensation as part of the head morphology (Figure 1D). Similarly, roselle extract has been used for SpragueDawley rat sperm staining, with comparable results to sperm stained with eosin [14]. The percentage of the defective heads after staining with black rice extract was closest to that obtained using rapid PAP (black rice extract rapid PAP $<$ SpermBlue $<$ DipQuick) (Figure 2). Because the basic procedure of black rice extract staining is similar to that of the rapid PAP method, except for the nuclear staining step, the black rice extract was used as a substitute for Harris hematoxylin. The fixation and/or dehydration steps of rapid PAP staining and the black rice extract procedure may also cause shrinkage of the spermatozoa [2]. DiffQuik causes sperm swelling and background staining, probably due to the difference between the osmolality of the semen and that of the fixatives and stains [5]. Ideally, stained spermatozoa should have dimensions as close to the spermatozoa in fresh semen as possible, as occurs with the SpermBlue staining method because the fixation and staining solution of SpermBlue is iso-osmotic in relation to semen [4].

Our statistical analysis indicated that the DipQuick staining method yielded a higher percentage of defective heads than was obtained by rapid PAP, black rice extract, and SpermBlue (Figures 2, 3E). Although DipQuick was mentioned in the 2010 WHO guideline as a rapid staining method, and it is widely used as a routine method for microscopic examination and for CASA [15], the results in this study indicate that using DipQuick could result in a statistically significant difference in sperm morphology evaluations. If a quick screening of spermatozoa is needed, the rapid test can be used. However, rapid PAP or SpermBlue should be used for assisted reproduction and diagnostic purposes $[5,16]$.

The Bland and Altman method was used to assess the degree of agreement. A small deviation was observed between the rapid PAP and black rice methods (Figure 3A). The value for the SpermBlue method fell between those of rapid PAP and DipQuick. Similar to the results shown in Figure 2, the highest mean differences were reported when the black rice extract was compared with the DipQuick stain. A possible reason for this result could be that DipQuick caused the head to swell, whereas the rapid PAP stain caused sperm head shrinking and SpermBlue had no significant effect on sperm head dimensions [5].

Passing-Bablok linear regression (Figure 3D, E) showed good agreement and a strong correlation between rapid PAP and black rice extract, and a moderate correlation between SpermBlue and black rice extract. In contrast, a weak correlation was observed between DipQuick and black rice extract. Therefore, the use of rapid staining may cause problems; for instance, a patient could be classified as subfertile by one laboratory and normal by another.

Mountain plots (Figure 4A) showed that the black rice extract stain corresponded with the rapid PAP better than the DipQuick stain, while the black rice extract and SpermBlue corresponded well with each other (Figure 4B). This finding indicates that black rice extract can be used instead of validated methods, such as rapid PAP and SpermBlue, for nuclear staining.

A previous study suggested that spermatozoa with head defects appear more or less normal in response to several factors, including semen sample preparation, the smearing technique, different staining method, the classification systems, and the technician's experience [10]. These factors could result in a decrease or an increase in the percentage of normal sperm morphology [5]. Furthermore, the present study revealed that the classification established by the 2010 WHO criteria is not suitable for all staining methods. Henkel et al. [16] found a significantly lower number of normal spermatozoa when a different staining method was used. Therefore, each spermatozoa stain should have established written criteria for sperm morphology evaluation for each staining method, accounting for factors such as semen sample preparation, the smearing technique, and the scoring or classification systems.

The present study has a few limitations. Most notably, the sample size was small, which might have limited the assessment of clinical usefulness. Further studies could also focus on comparing the staining techniques in clinical samples. In conclusion, as a novel nuclear stain substitute for hematoxylin, black rice extract provides several advantages such as ease of preparation, local availability, and favorable nuclear staining properties. The authors recommend black rice extract as an alternative stain in routine sperm staining for sperm head morphology evaluation.

\section{Conflict of interest}

No potential conflict of interest relevant to this article was reported.

\section{ORCID}

Sirinart Chomean https://orcid.org/0000-0003-2310-2934

Chollanot Kaset https://orcid.org/0000-0002-2110-6918

\section{References}

1. World Health Organization. WHO laboratory manual for the examination and processing of human semen. 5th ed. Geneva: World Health Organization; 2010.

2. World Health Organization. WHO laboratory manual for the examination of human semen and semen-cervical mucus interaction. 4th ed. Cambridge: Cambridge University Press; 1999.

3. Kruger TF, Ackerman SB, Simmons KF, Swanson RJ, Brugo SS, Acosta AA. A quick, reliable staining technique for human sperm 
morphology. Arch Androl 1987;18:275-7.

4. van der Horst G, Maree L. SpermBlue: a new universal stain for human and animal sperm which is also amenable to automated sperm morphology analysis. Biotech Histochem 2009;84:299308.

5. Maree L, du Plessis SS, Menkveld R, van der Horst G. Morphometric dimensions of the human sperm head depend on the staining method used. Hum Reprod 2010;25:1369-82.

6. Dapson R, Horobin RW, Kiernan J. Hematoxylin shortages: their causes and duration, and other dyes that can replace hemalum in routine hematoxylin and eosin staining. Biotech Histochem 2010;85:55-63.

7. Bhattacharjee $P$, Singhal RS, Kulkarni PR. Basmati rice: a review. Int J Food Sci Technol 2002;37:1-12.

8. Pengkumsri N, Chaiyasut C, Saenjum C, Sirilun S, Peerajan S, Suwannalert $P$, et al. Physicochemical and antioxidative properties of black, brown and red rice varieties of northern Thailand. Food Sci Technol 2015;35:331-8.

9. Benard SA. Iron-Roselle: a progressive nuclear stain substitute for hematoxylin. J Histotechnol 2008;31:57-9.

10. Wang Y, Yang J, Jia Y, Xiong C, Meng T, Guan H, et al. Variability in the morphologic assessment of human sperm: use of the strict criteria recommended by the World Health Organization in 2010. Fertil Steril 2014;101:945-9.

11. Koo TK, Li MY. A guideline of selecting and reporting intraclass correlation coefficients for reliability research. J Chiropr Med 2016;15:155-63.

12. Saenguthai K, Chomean S, Kaset C. The evaluation of Oryza sativa $L$ (Black rice) extracts for detection of spermatozoa on the clothing and vaginal swab samples. Leg Med (Tokyo) 2018;35: 91-7.

13. Chomean S, Nantabut M, Kongtia W, Saenguthai K, Kaset C. Evaluation of natural dyes for human spermatozoa morphology assessment. Acta Histochem 2019;121:227-33.

14. Bassey RB, Osinubi AA, Oremosu AA. Staining effect of Hibiscus sabdariffa extract on sperm cell morphology of Sprague-Dawley rats. J Histotechnol 2012;35:110-3.

15. Coetzee K, Bermes N, Krause W, Menkveld R. Comparison of normal sperm morphology outcomes from two different computerassisted semen analysis systems. Andrologia 2001;33:159-63.

16. Henkel R, Schreiber G, Sturmhoefel A, Hipler UC, Zermann DH, Menkveld R. Comparison of three staining methods for the morphological evaluation of human spermatozoa. Fertil Steril 2008;89:449-55. 